\title{
Benefits of telemedicine in diagnosis of herpes zoster ophthalmicus with timely management and follow-up
}

\section{Udaya Kiran Koduri}

\author{
Medilab Clinic, Hyderabad, India
}

Corresponding author: Udaya Kiran Koduri, MD, E-mail: ukiran927@gmail.com

Sir,

Herpes Zoster is caused by the viral reactivation of Varicella Zoster virus, that enters the cutaneous nerve endings during an earlier episode of chicken pox and travels to the Dorsal Root Ganglia and remains latent. It is characterized by the occurrence of vesicular lesions grouped along the Single Dermatome innervated by Dorsal Root of Sensory Ganglion.

Herpes Zoster Ophthalmicus (HZO) is involvement of the ophthalmic division of the fifth cranial nerve. It is the second most common type of Herpes Zoster (HZ), after Thoracic Zoster [1].

We present here, a case of Herpes Zoster in a young girl involving Supraorbital, Maxillary and Mandibular of the Trigeminal nerve.

Timely diagnosis of HZO was made. The lesion on the tip of the nose (Hutchinson's sign) alerted the involvement of Nasociliary nerve and to arrange immediate Ophthalmology consultation. TeleDermatology helped in reducing waiting time, fast delivery of care and prevention of visual disability [2].

The patient was monitored, motivated and treated, that could happen because of tele- dermatology. Dramatic improvement in the patient was observed in a very short time. The use of tele-dermatology, especially in times of the COVID - 19 pandemic is invaluable.

The patient was a girl aged 12-years, presented through telemedicine with 3 days of lesions on the face mimicking acne. On close examination, there were a few Vesicular Lesions among multiple lesions and there was involvement of tip of the nose. Patient also complained of eye pain. This helped us to consider HZO. Prompt immediate treatment with anti-virus was initiated. It was possible to do continuous monitoring with the help of Tele-Medicine and without the patient having to leave the house in COVID era.

Patient was advised to consult an Ophthalmologist for further evaluation. As it was essential, for examination of the eye parameters, patient had to attend in person. This one visit was not through Tele-Medicine.

Patient was informed on day-l of treatment that the condition may initially worsen under proper adequate treatment with a graphic representation (Fig. 1) and then decrease later. And this happened in our case, as seen in the six consecutive photographs as indicated below with date and time of the photographs by the patient, which are presented to illustrate the natural progression of disease under proper adequate treatment. This helped in avoiding panic to the patient. The lesions as seen in the pictures became aggressive in two days which means, the eye could have been affected more but for the immediate treatment given.

Patient could get treatment from home by consulting online through out, thereby reducing the risk of venturing to a clinic in COVID situation. Except for one visit to the Ophthalmologist which was inevitable.

Ocular Manifestations - Ocular manifestations affect about $50 \%$ of patients with $\mathrm{HZO}$ and can be isolated. This proportion reaches $80 \%$ in case of appearance of the Hutchinson Sign. The latter reflects the involvement of

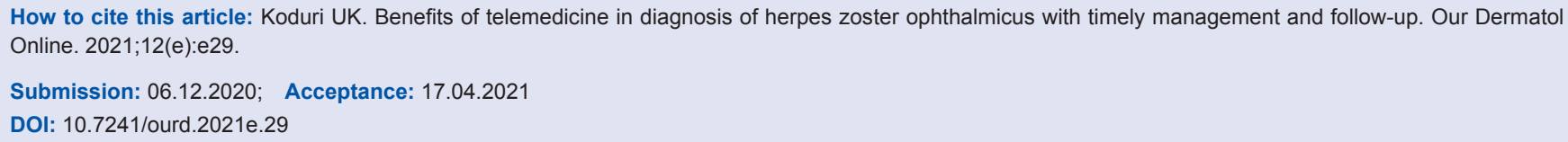



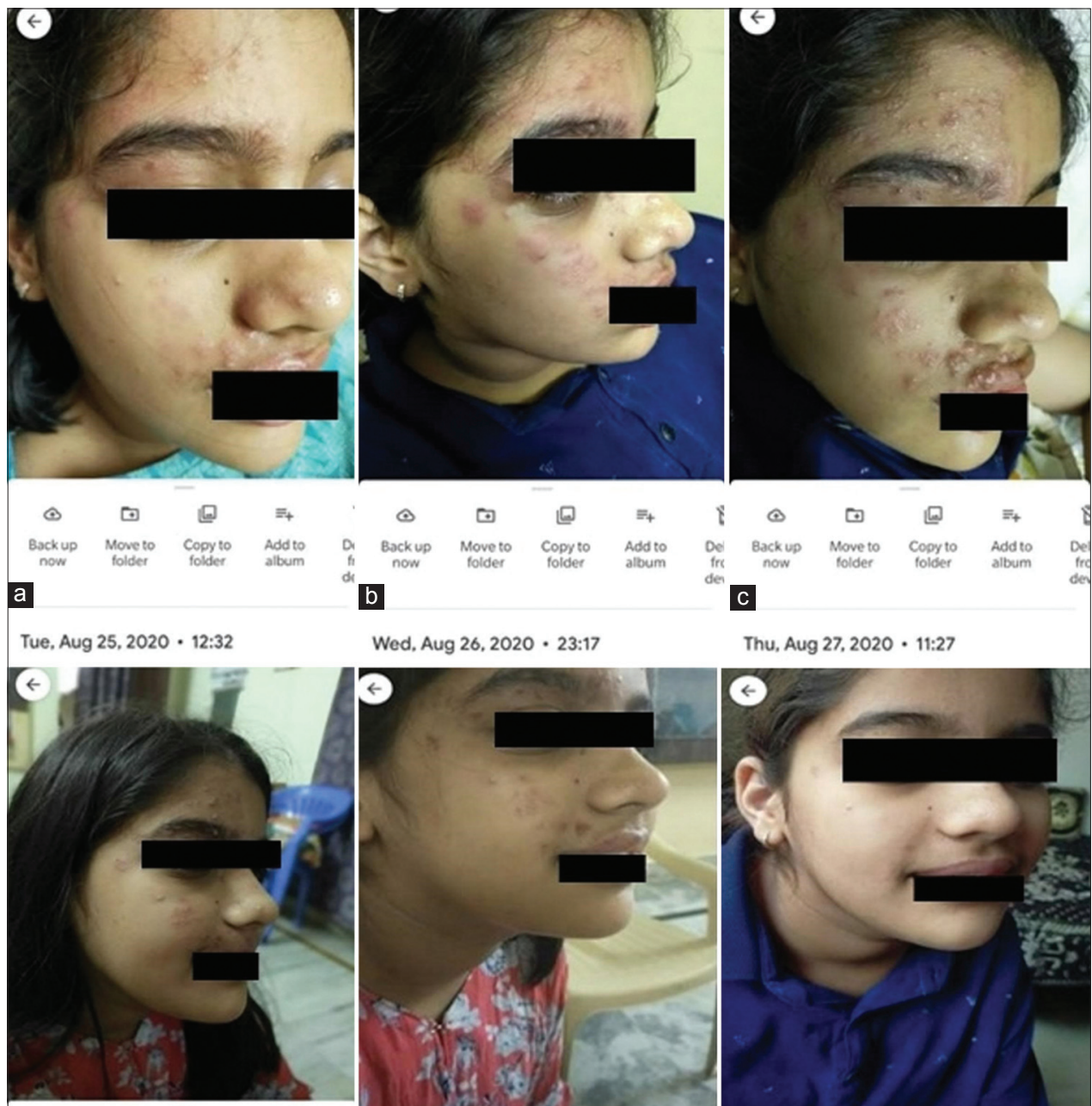

Wed, Aug 26, 2020 - 23:17

Thu, Aug 27, $2020 \cdot 11: 27$
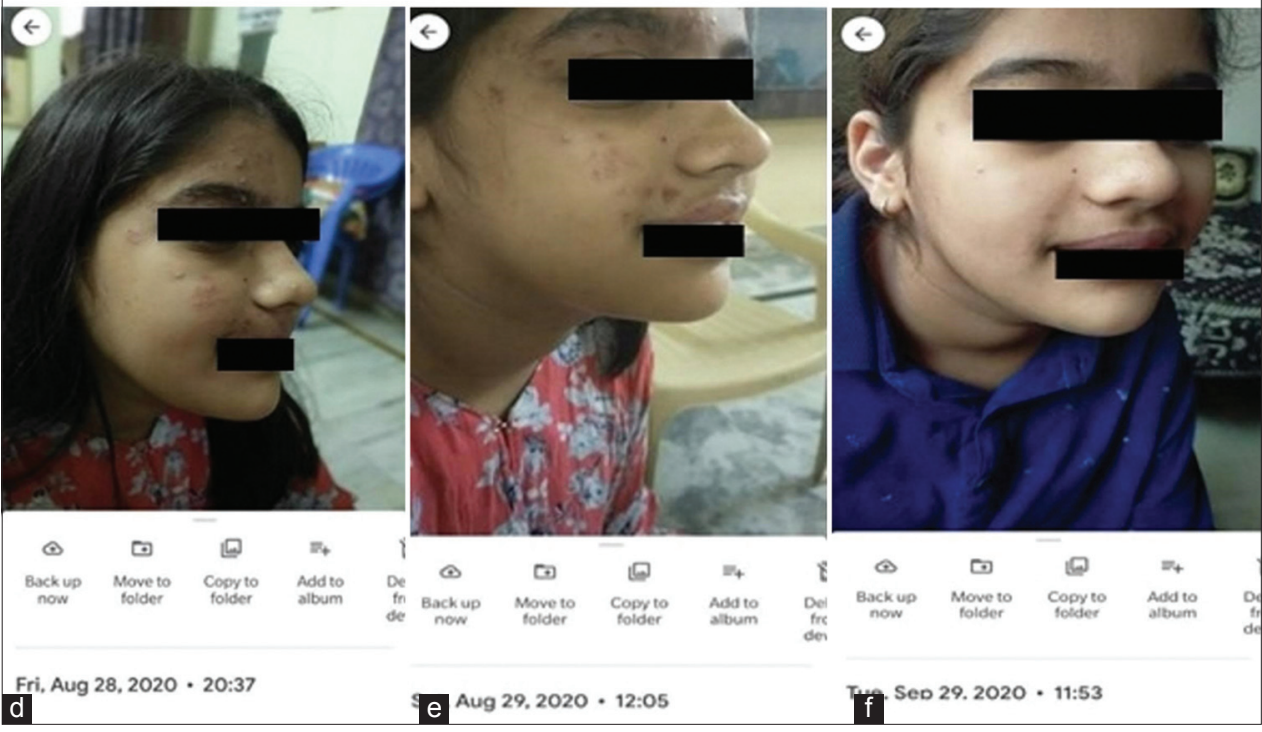

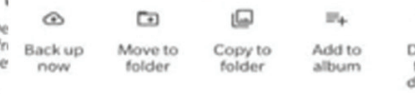

Se Aug 29.2020 - 12:05

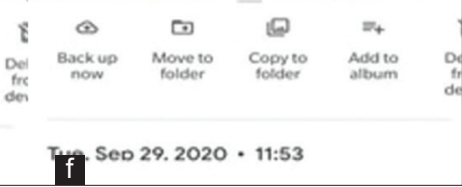

Figure 1: (a) Image shared by the patient at the start of the treatment on August 05, 2020. (b) Image shared by the patient during the course of the treatment on August 26, 2020. (c) Image shared by the patient during the course of the treatment on August 27, 2020. (d) Image shared by the patient during the course of the treatment on August 28, 2020. (e)Image shared by the patient during the course of the treatment on August 29, 2020. (f) Image shared by the patient post completion of the treatment on September 29, 2020.

the nasociliary branch and is characterized by eruption on the side and on the tip of the nose [3].

Eye examination showed that there was congestion generalized in Conjunctiva. Corneal sensation was reduced, Iris anterior chamber, Pupil, lens, Fundus and extra ocular movements were normal.

Immediate treatment was instituted after counselling about the treatment. The patient was advised Valacyclovir $1000 \mathrm{mg}, 3$ times a day for 7 days along with adequate fluid intake. For relief of pain, patient was given Pregabalin, Tremedol $25 \mathrm{mg}$ and Gabapentin 6\%W/W+Lidocaine 5\%W/W smooth locally.

\section{Eye treatment}

As per Ophthalmologists advice, Polyethylene glycol 400 0.4\% + Propylene glycol $0.3 \%$ five times a day and Acyclovir eye ointment a day was prescribed to the patient.

All the pictures were taken by the patient and shared with the doctor, during the course of the treatment helping in regular monitoring have been presented $(1 \mathrm{a}-\mathrm{lf})$.

In this case, Telemedicine gave the scope of reducing waiting time, providing correct diagnosis, enabling 




Figure 2: Graphic representation of the course of the Herpes Zoster Ophthalmicus.

faster delivery of care, enabling tele based access to monitor the patient's condition, counselling, management, monitoring with good end result.

\section{Key Takeaways}

- The importance of (Hutchinson's sign) involvement of the tip of nose is emphasized.

- Counselling the patient with regard to the possibility of worsening of the visible symptoms while under appropriate and adequate treatment, should be given with the help of a graphic representation (Fig. 2).
- Prompt referral to Ophthalmologist is necessary.

- The consultant and the patient should be conscious of associated potential Vision threatening complications and the need for prompt treatment to protect from visual complications.

\section{Consent}

The examination of the patient was conducted according to the principles of the Declaration of Helsinki.

The authors certify that they have obtained all appropriate patient consent forms, in which the patients gave their consent for images and other clinical information to be included in the journal. The patients understand that their names and initials will not be published and due effort will be made to conceal their identity, but that anonymity cannot be guaranteed.

\section{REFERENCES}

1. Mishra A, Baranwal VK, Patra VK, Srivastava VK. An interesting case of herpes zoster ophthalmicus. Med J DY Patil Univ. 2013;6:289-91.

2. Janniger CK, Eastern JS, Hospenthal DR, Moon JE. Herpes zoster treatment \& management. Emedicine Medscape- article/1132465treatment

3. Rousseau A, Bourcier T, Colin J, Labetoulle M. Herpes zoster ophthalmicus_-diagnosis and management. US Ophthal Rev. 2013;6:119-24.

Copyright by Udaya Kiran Koduri. This is an open access article distributed under the terms of the Creative Commons Attribution License, which permits unrestricted use, distribution, and reproduction in any medium, provided the original author and source are credited.

Source of Support: Nil, Conflict of Interest: None declared. 